\title{
Adverse Reactions to Anthrax Immunisation in a Military Field Hospital
}

\author{
S C Hayes, M J World
}

\author{
ABSTRACT \\ Objective \\ To determine the outcome of anthrax \\ immunisation.
}

\section{Methods}

Adverse reactions (occurrence, nature, severity and incapacity) and immune responses to a voluntary programme of anthrax immunisation at $0,3,6$, and 24 weeks were monitored by questionnaire and voluntary blood sampling in 129 members, including 24 immunised 7 years previously (immunes), of a military field hospital alerted for possible deployment.

\section{Results}

Follow-up was complete in $85 \%$. Ninety-eight $(76 \%)$ received the first anthrax immunisation. Uptake was greater $(p=0.015)$ in immunes. Initial prevalence of adverse reaction was $63 \%$. Subsequent uptake and adverse reaction dwindled significantly $(\mathrm{p}<0.001)$. Only $28 \quad(22 \%)$ were immunised at 24 weeks. Proportions reporting adverse reactions following the initial immunisation were greater in immunes $(p=0.046)$ and officers $(p=0.02)$. There was no significant $(p=0.36)$ correlation between uptake of immunisation and prevalence of adverse reaction. Antecedent adverse reaction did not reduce the proportion of participants accepting immunisation subsequently. The nature of adverse reactions (47\% local, $24 \%$ systemic and $27 \%$ both ) and severity were the same throughout. Forty-five percent of adverse reactions caused incapacity. Seventy-four percent of these had pain in the injected arm ( \pm systemic symptoms) which prevented lifting or driving for 48 hours in $63 \%$. Immune responses were greater in immunes.

Lt Col MJ World BSc

MD FRCP RAMC

Professor of Military

Medicine,

Royal Defence

Medical College,

Fort Blockhouse,

Gosport, Hants,

PO12 2AB

E-Mail:

106201.1307@compuserve.com

\section{Conclusions}

It was concluded that anthrax immunisation results in a higher than expected prevalence of adverse reaction with initial incapacity of military significance affecting $18 \%$. Greater immune responses may increase adverse reaction but this does not affect acceptance of anthrax immunisation.
Poor completion rates necessitate development of a new anthrax immunisation strategy.

\section{Introduction}

Voluntary Immunisation

In March 1998, a military field hospital was alerted for possible deployment and 129 members were listed to receive intramuscular immunisations with anthrax vaccine at $0,3,6$ and 24 weeks. This was voluntary in response to concerns of Gulf War 1990/1991 veterans that, amongst others, anthrax immunisation could cause "Gulf War Syndrome”.

\section{Anticipated prevalence of side effects}

Between 1976 and 1998, the Committee on the Safety of Medicines (CSM) received 19 reports of adverse reactions at a time when about 2200 doses of anthrax vaccine were issued annually. Only $10-15 \%$ of serious reactions may be reported to the CSM. Hence, with a possible total of 516 doses being administered, only one or two adverse reactions were expected and sick parade was considered satisfactory surveillance. However, when several personnel who had not attended sick parade mentioned having had significant adverse reactions following the first immunisation, a study was rapidly devised to elicit reports and to assess the relation to the immune response following each immunisation.

\section{Methods}

Data acquisition

Personal information and any previous immunisation were recorded. After each immunisation, a questionnaire (copies available from MJW) about any adverse reaction (nature, severity and associated incapacity) was provided. A copy was sent to absentees. Subject to written informed consent, a $5 \mathrm{ml}$ blood sample was taken and serum was stored at $-80^{\circ}$ Celsius for enzyme-linked immunosorbent assay (ELISA) for IgG to recombinant protective antigen.

\section{Statistical Analysis}

When sample variances were homoscedastic by F-test, the significance of differences between two mean values was assessed using Student's $t$-test and otherwise of medians by Mann-Whitney U-test. 
Differences between three or more mean values were assessed by one-way ANOVA. The significance of differences in proportions in contingency tables was assessed by $\chi^{2}$-test or Fisher's exact test as appropriate. Where trends were suspected in contingency tables, $\chi^{2}$-test for trend was applied. Linear correlation between continuous variables was assessed by the method of least squares and the significance of the correlation coefficient assessed by Student's $t$-test. Statistical significance was considered to exist in all circumstances when $\mathrm{p}<0.05$.

\section{Results}

Completeness of response

Follow-up was complete in $110(85 \%)$. Of the remaining 19 not contacted, only 4 received the first anthrax immunisation and personal details of 4 were available from staff records.

\section{Personnel}

Fifty-eight (45\%) were permanent staff reinforced by $56(43 \%)$ from a military hospital and $15(12 \%)$ from other units. Demography is shown in Table 1 . All but 5 (4\%) of 114 were caucasian. Twenty-four (19\%) had anthrax immunisation 7 years previously (immunes) during the Gulf War (1990/91). Anthrax immunisation status of $25(19 \%)$ was undetermined.

\begin{tabular}{lllll}
\hline & Total & Males & Females & $\begin{array}{l}\text { Gender } \\
\text { Unrecorded }\end{array}$ \\
\hline Officers & $47(36 \%)$ & $28(59 \%)$ & $17(36 \%)$ & $2(4 \%)$ \\
Mean Age (yr) & 35.61 & 38.07 & 31.19 & \\
( \pm SD;n) & $(+-6.67 ; 42)$ & $+-6.53 ; 27)$ & $(+-3.82 ; 15)$ & \\
Other Ranks & $82(64 \%)$ & $53(65 \%)$ & $21(26 \%)$ & $8(10 \%)$ \\
Mean Age (yr) & 29.19 & 29.21 & 29.13 & \\
( \pm SD;n) & $(+-4.66 ; 73)$ & $+-4.93 ; 52$ & $(+-3.81 ; 21)$ & \\
Total & $129(100 \%)$ & $81(63 \%)$ & $38(29 \%)$ & $10(8 \%)$ \\
\hline
\end{tabular}

Table 1. Demography of the military field hospital.

The proportions of males and females were not statistically significantly different between the Officers and Other Ranks ( $\chi^{2}$ [Yates' continuity corrected] $=0.7461, d f=1 ; p$ $=0.39)$. The mean age of Officers was higher than that of Other Ranks and this difference was statistically significant (variances were heteroscedastic $F=2.045, D F n=41$, $D f d=72, \quad p=0.0039 ; \quad M a n n-W h i t n e y \quad U=660.5$, $p<0.0001)$. This difference in age was due to the higher mean age of Male Officers when compared with that of Male Other Ranks. This difference was statistically significant (variances were heteroscedastic $F=1.788$, $D F n=26, D f d=51, p=0.038 ;$ Mann-Whitney $U=190$, $p<0.0001)$ Mean ages of Female Officers and Female Other ranks were not statistically significantly different $(t=1.555 d f=34, p>0.05)$

\begin{tabular}{llll}
\hline $\begin{array}{l}\text { Date of anthrax } \\
\text { immunisation } \\
\text { (whs) }\end{array}$ & $\begin{array}{l}\text { No (\%) who did } \\
\text { receive anthrax } \\
\text { immiunisation }\end{array}$ & $\begin{array}{l}\text { No (\%) who did } \\
\text { not receive anthrax } \\
\text { immiunisation }\end{array}$ & Total \\
\hline 0 & $98(76 \%)$ & $31(24 \%)$ & 129 \\
3 & $73(57 \%)$ & $56(43 \%)$ & 129 \\
6 & $41(32 \%)$ & $88(68 \%)$ & 129 \\
24 & $28(22 \%)$ & $101(78 \%)$ & 129 \\
& 240 & 276 & 516 \\
\hline
\end{tabular}

Table 2. The proportions of subjects who did and did not receive anthrax immunisation at each stage of immunisation were statistically significantly different with a highly statistically significant trend towards reduced receipt of anthrax immunisation as time passed $\left(\chi^{2}=93.42, d f=3\right.$; $p<0.0001 ; \chi^{2}$ for trend $=91.42, d f=1 ; p<0.0001$ )
Acceptance of anthrax immunisation

This is shown in Table 2. Cumulative figures were virtually the same (data not shown). Uptake dwindled significantly. As the proportion of permanent staff accepting immunisation decreased from $44 \%$ to $25 \%$, that of reinforcements increased $(p=0.015$. Data not shown)

Factors affecting acceptance of the first anthrax immunisation at 0 weeks.

The proportion of immunes accepting initial immunisation $(23 / 24)$ was greater than that of the naive $(75 / 105 ; \mathrm{p}=0.015$. Difference in proportions $=0.24[95 \% \mathrm{CI}$ $0.12-0.38] ;$ odds ratio $=9.2[95 \%$ CI 1.19 $-71.2])$.

Prevalence of adverse reaction

This dwindled with time and is shown in Table 3.

\begin{tabular}{llll}
\hline $\begin{array}{l}\text { Date of anthrax } \\
\text { immunisation } \\
\text { (whs) }\end{array}$ & $\begin{array}{l}\text { No (\%) who did } \\
\text { have an adverse } \\
\text { reaction to anthrax } \\
\text { immiunisation }\end{array}$ & $\begin{array}{l}\text { No (\%) who did } \\
\text { not have an adverse } \\
\text { reaction to anthrax }\end{array}$ & Total \\
\hline 0 & $62(63 \%)$ & $36(37 \%)$ & 98 \\
3 & $14(19 \%)$ & $59(81 \%)$ & 73 \\
6 & $14(34 \%)$ & $27(66 \%)$ & 41 \\
24 & $7(25 \%)$ & $21(75 \%)$ & 28 \\
& 97 & 143 & 240 \\
\hline
\end{tabular}

Table 3. The proportions of subjects who did and did not report an adverse reaction to anthrax immunisation at each stage of the schedule were statistically significantly different with a highly statistically significant trend towards reduced prevalence of adverse reaction as time passed $\left(\chi^{2}=38.35\right.$, $d f=3 ; p<0.0001 ; \chi^{2}$ for trend $=18.67, d f=1 ; p<0.0001$ )

Factors affecting report of an adverse reaction to anthrax immunisation at 0 weeks

Adverse reaction in immunes (19/23) exceeded that in the naive $(43 / 75 ; \mathrm{p}=0.046$. Difference in proportions $=0.25[95 \% \mathrm{CI}$ $0.16-0.48$; odds ratio $=3.54[95 \% \mathrm{CI}$ 1.10 - 11.42]). Gender had no effect $(p=0.66$. Data not shown) Officers had increased risk $(p=0.02$. Data not shown) but were older. However, when all subjects were stratified by quartiles according to age, there was no significant trend for the prevalence of adverse reaction to increase with age $(p=0.44$. Data not shown)

Effect of an adverse reaction to antecedent anthrax immunisation on the prevalence of adverse reaction to a subsequent immunisation. Antecedent adverse reactions were found to affect the prevalence of adverse reaction to subsequent anthrax immunisation only at 6 weeks when the proportion of those reporting adverse reactions at 0 and 3 weeks who also had an adverse reaction at 6 weeks $(7 / 12)$ was greater than that $(0 / 7)$ who did not report adverse reactions at 0 and 3 weeks $(\mathrm{p}=0.02$. Difference in proportions $=$ 0.58 [95\% CI $0.30-0.86]$; odds ratio $=\infty$ ). 
Lack of correlation between acceptance of anthrax immunisation and prevalence of adverse reaction

No correlation between acceptance of anthrax immunisation and prevalence of adverse reaction was found $(r=0.64$; $\mathrm{p}=0.36$ ).

Effect of a preceding adverse reaction on the acceptance of a subsquent immunisation

Antecedent adverse reaction was found to affect subsequent immunisation only once and then favourably. The proportion of those reporting adverse reactions at 0 weeks and 3 weeks who received immunisation at 6 weeks $(12 / 13)$ was greater than that $(7 / 22)$ of those who did not report adverse reactions at 0 and 3 weeks and had immunisation at 6 weeks $(p=0.001$. Difference in proportions $=0.453[95 \% \mathrm{CI}$ $0.36-0.84]$; odds ratio $=25.71[95 \% \mathrm{CI}$ $2.74-239.51])$

Classification of diverse reactions and prevalence following each immunisation

The distribution between three mutually exclusive categories of adverse reaction did not change at any stage $(\mathrm{p}=0.55)$. Overall, $47 \%$ experienced local (pain at the injection site) reactions, $24 \%$ systemic (mainly influenza-like) reactions and $27 \%$ both (data not shown).

\section{Severity of adverse reactions}

This did not change during the immunisation schedule $(p=0.75$. Data not shown). Overall, $29 \%$ of reactions were classified as mild, $49 \%$ moderate and $21 \%$ severe.

\section{Incapacity}

The proportion of personnel reporting incapacity as a result of adverse reactions did not differ at any stage $(p=0.75$. Data not shown). Whatever the adverse reaction, $45 \%$ were incapacitated $(p=0.09$. Data not shown). The distribution of incapacitated personnel between the three categories of adverse reaction did not change during the immunisation schedule $(p=0.76$. Data not shown). Twenty-seven (63\%) of the 43 reports of incapacity mentioned the duration, the distribution of which was the same throughout the schedule $(\mathrm{p}=0.1$, Data not shown). Overall, the duration of the majority $(63 \%)$ of incapacitating adverse reactions (mainly pain at the site of injection preventing lifting or driving) did not exceed 48 hours. The maximum duration of incapacity was 120 hours.

\section{Immune responses}

Concentrations of IgG to recombinant protective antigen at different times are shown in the figure. Wide variation is apparent. Mean antibody levels were generally higher in immunes being statistically significant at 3 weeks $(\mathrm{p}=0.006)$ and 24 weeks $(\mathrm{p}=0.006)$. A trend towards a rise in concentration was seen in the naive but mean values were not statistically significant different $(p=0.59)$.

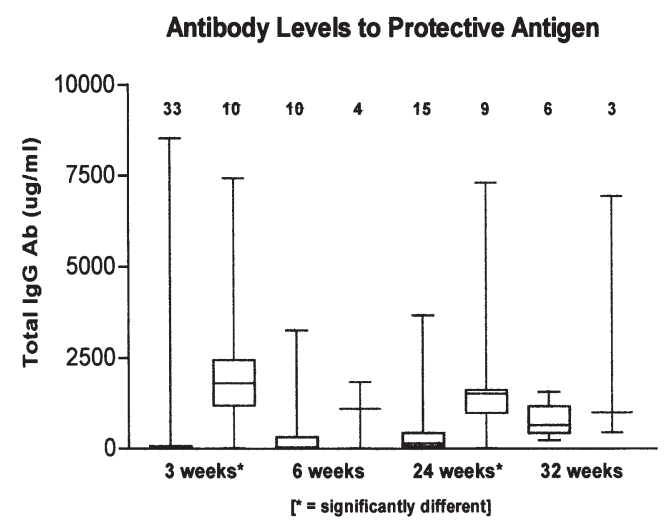

Serum concentration of total IgG antibody to recombinent protective antigen was measured by enzyme-linked immunosorbent assay (please see acknowledgements). Box and whiskers plots showing median values, quartiles and ranges are shown for those immunised 7 years previously (right) and the naive (left) at each time before reimmunisation and also at 32 weeks. The number of voluntary blood samples on which data are based are shown above each box and whiskers plot.

\section{Discussion}

Protection against anthrax

Anthrax spores are a potent biological weapon (1). Fatal pulmonary anthrax results when sufficient spores are inhaled unless some effective preventive measure is taken (2). Immunisation is one method (3). Pure recombinant protective antigen administered with Ribi adjuvant to experimental animals is encouraging (4) but has yet to be assessed in man. Although the old vaccine is considered safe, the number of adverse reactions and incapacity reported by a military medical unit was unexpected.

\section{Completion of immunisation schedule}

Initial acceptance of immunisation (76\%) was comparable to that (78\%) reported in American mill workers not withdrawn from a clinical trial (5). In the present study, only $27(21 \%$ of those initially listed) completed the immunisation schedule. A lower fall in uptake was found when civilian volunteers were immunised (6). Continuing exposure to anthrax spores and a differing immunisation schedule might explain this difference. Decreased motivation could result when the threat of anthrax subsided. Non-availability of vaccine when reinforcement personnel returned to parent units was unlikely as reinforcements constituted an increasing proportion of those receiving subsequent immunisations. Adverse reactions were not the cause of this. Others $(5,6)$ found an increasing proportion had adverse reactions as time passed. However, adverse reactions decreased in the present study with those affected being undeterred from accepting subsequent immunisation. 


\section{Previous immunisation}

Those immunised against anthrax 7 years previously were more willing to undergo immunisation than naive subjects and were more likely to report adverse reactions after the first immunisation of a new series. Recent evidence (7) suggested recipients of biological or multiple immunisations were more likely to satisfy the Centers for Disease Control and Prevention definition of "Gulf War syndrome" (8). Nevertheless, the small group of British GulfWar veterans in the present study were not deterred from accepting anthrax re-immunisation and, presumably, had not associated previous immunisation with unduly adverse reactions or persisting symtoms. A previous report (5) mentioned two mill workers processing imported goat hair who were inadvertently immunised against anthrax when they had had clinical cutaneous anthrax 7 and 14 years previously. Both experienced severe local reactions at 24 and 48 hours after immunisation indicating that adverse reactions can occur in those reexposed to anthrax antigens many years later.

\section{Prevalence of adverse reactions}

The overall prevalence of adverse reactions $(40 \%)$ was similar to that $(35 \%)$ reported previously (5) but then the great majority of reactions were local with just 2 subjects (a prevalence of $0.2 \%$ ) experiencing systemic reactions (see below).

Nature and severity of adverse reactions and associated incapacity

The distribution of adverse reactions (local, systemic and local with systemic) remained constant. Previously a decreasing prevalence of local reaction and an increasing prevalence of local with systemic reaction were reported (6). Distribution of severity between categories (mild, moderate and severe) remained constant with only a minority $(21 \%)$ reporting severe symptoms. Of the 95 experiencing any type of adverse reaction at any time (the prevalence of adverse reaction decreased with time), 43 ( $45 \%$ ) reported associated incapacity and of these, 27 gave the duration of incapacity. This lasted not more than 48 hours in the majority $(17=63 \%)$. Thus, $18 \%$ of those receiving the initial immunisation were incapacitated for 48 hours but only $7 \%$ of those receiving the final immunisation. In most $(74 \%)$, pain at the site of injection prevented lifting or driving for 48 hours. Previously, it was claimed that no incapacity occurred after anthrax immunisation (6).

Relationship between adverse reaction and immune reponse

Darlow, Belton and Henderson (6) found no consistent relationship between the severity of reaction and the toxin- neutralising antibody titre assessed by bioassay. The present study using a more sophisticated method to estimate antibody concentration supports this.

What prevented acceptance of immunisation? No positive evidence has emerged to account for the failure to accept immunisation. Partly this was due to the omission of systematic enquiry whether members of the field hospital staff feared the risk of "Gulf War Syndrome" arising following anthrax immunisation and of why those failing to complete the immunisation schedule did so. However, the proportion of officers $(38 / 47=81 \%)$ accepting initial immunisation was not significantly different from that of other ranks $(60 / 82=$ $73 \%$ ) suggesting that seniority did not affect acceptance. It was mentioned above that reinforcements were more likely to complete the immunisation schedule but the proportion of these accepting initial immunisation $(55 / 73=75 \%)$ was not significantly different from that of the permanent staff of the field hospital (43/56 $=77 \%)$. This suggests that whilst higher qualification may result in more likely completion of the immunisation schedule, it has no affect on initial acceptance. The facts that previous anthrax immunisation did not deter re-immunisation and that antecedent adverse reactions during the present schedule of immunisation did not prevent subsequent immunisation have been discussed already. Diminishing likelihood of deployment with the passage of time cannot be discounted as a cause of failure to complete the immunisation schedule.

Measures to improve the completion of the immunisation schedule

Anthrax immunisation is essential for protection. If compulsory immunisation for military personnel is unacceptable, a superior antigen inducing long lasting immunity following a single immunisation is required. Improvement in voluntary acceptance of this could result if potential recipients are shown the results of animal experiments which demonstrate dramatically the benefit of immunisation (3). Side effects are unlikely to deter subjects from receiving immunisation provided these do not exceed the magnitude of those reported here.

\section{Ackowledgments}

The authors are grateful to the members of 33 Field Hospital RAMC without whose co-operation this study would not have been possible and to Messrs TJ Townend and LWJ Baillie of Chemical \& Biological Defence Sector, DERA Porton Down, Salisbury who kindly assayed antibody concentrations. 
Footnote:

Data not shown were made available for the scrutiny of reviewers and are available on request.

\section{References}

1. Pile JC, Malone JD, Eitzen EM, Friedlander AM. Anthrax as a potential biological warfare agent. Arch Int Med 1998; 158: 429-434

2. Penn CC, Klotz SA. Anthrax pneumonia. Semin Respir Infect 1997; 12: 28-30

3. Freidlander AM, Welkos SL, Pitt MLM, Ezzell JW, Worsham PL, Rose KJ et al. Postexposure prophylaxis against experimental inhalation anthrax. F Infect Dis 1993; 167: 1239-1242

4. Miller J, McBride BW, Manchee RJ, Moore P, Baillie LWJ. Production and purification of recombinant protective antigen and protective efficacy against Bacillus anthracis. Lett Appl Microbiol 1998; 26: 56-60

5. Brachman PS, Gold H, Plotkin S, Fekety FR, Werrin $M$, Ingraham NR Field evaluation of a human anthrax vaccine. Am F Public Health 1962; 52: $632-645$

6. Darlow HM, Belton FC, Henderson DW. The use of anthrax antigen to immunise man and monkey. Lancet 1956; II: 476-478

7. Unwin C, Blatchley N, Coker W, Ferry S, Hotopf $M$, Hull L et al. Health of UK servicemen who served in Persian GulfWar. Lancet 1999; 353: 169178

8. Fukuda K, Nisenbaum R, Stewart G, Thompson WW, Robin L, Washko RM et al. Chronic multisymptom illness affecting air force veterans of the gulf war. $\mathcal{F A M A}$ 1998; 280: 981-988

9. Ismail K, Everitt B, Blatchley N, Hull L, Unwin C, David A et al. Is there a GulfWar syndrome? Lancet 1999; 353: 179-182 